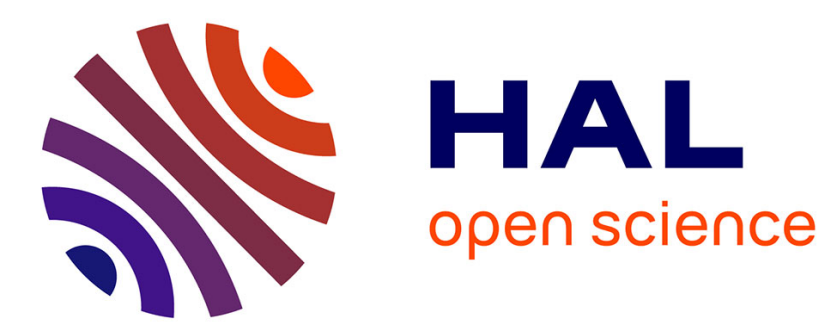

\title{
Quality-driven and real-time iris recognition from close-up eye videos
}

\author{
Valérian Némesin, Stéphane Derrode
}

\section{To cite this version:}

Valérian Némesin, Stéphane Derrode. Quality-driven and real-time iris recognition from close-up eye videos. Signal, Image and Video Processing, 2016, 10 (1), pp.153-160. 10.1007/s11760-014-0720-x . hal-01276600

\section{HAL Id: hal-01276600 \\ https://hal.science/hal-01276600}

Submitted on 20 Feb 2016

HAL is a multi-disciplinary open access archive for the deposit and dissemination of scientific research documents, whether they are published or not. The documents may come from teaching and research institutions in France or abroad, or from public or private research centers.
L'archive ouverte pluridisciplinaire HAL, est destinée au dépôt et à la diffusion de documents scientifiques de niveau recherche, publiés ou non, émanant des établissements d'enseignement et de recherche français ou étrangers, des laboratoires publics ou privés. 
Noname manuscript No.

(will be inserted by the editor)

\section{Quality-driven and real-time iris recognition from close-up eye videos}

\author{
Valérian Némesin . \\ Stéphane Derrode
}

Received: date / Accepted: date

\begin{abstract}
This paper deals with the computation of robust iris templates from video sequences. The main contribution is to propose (i) optimal tracking and robust detection of the pupil, (ii) smart selection of iris images to be enrolled, and (iii) multi-thread and quality-driven decomposition of tasks to reach real-time processing. The evaluation of the system was done on the Multiple Biometric Grand Challenge dataset. Especially we conducted a systematic study regarding the fragile bit rate and the number of merged images, using classical criteria. We reached an equal error rate value of $0.2 \%$ which reflects high performance on this database with respect to previous studies.
\end{abstract}

Keywords Iris biometrics - Quality-driven iris-code fusion · Real-time iris enrollment · MBGC dataset evaluation

\section{Introduction}

Iris biometric is generally accepted as one of the most reliable method for human identification, due to the high variability in iris texture and its stability over time [11]. However most existing iris recognition systems impose strong acquisition constraints which limit its widespread for largepublic applications: users have to present their eye at close

\section{Némesin}

Aix Marseille Université, Centrale Marseille, CNRS, Institut Fresnel, UMR 7249, 13013 Marseille, France

Tel.: +03349128 8328

E-mail: valerian.nemesin@centrale-marseille.fr

S. Derrode

École Centrale de Lyon, CNRS, LIRIS, UMR 5205, 69134 Lyon, France

Tel.: +033472186445

E-mail: stephane.derrode@ec-lyon.fr distance (about $0.3 \mathrm{~m}$ from the device) to ensure good focusing and quality of captured images. The iris biometric research community is now interested in setting less constrained environments, and in designing systems that are able to capture iris at distance and on the move, which can help improving user acceptability and collectability [10]. Additionally to problems encountered in still-device systems, videobased ones further face motion blur and lack of resolution. A solution is to adapt iris segmentation methods [22,7] for noisy images or non-ideal environments to the video context.

Nevertheless, in video-based systems, one aims at exploiting numerous views of the same iris to get one iris-code that is more robust (in term of recognition performance) than the iris-code obtained from the best-quality iris image in the video. Two main families of methods for merging iris-codes from close-up eye videos were proposed.

The first family considers the direct fusion of gray-level pixels of polar iris texture to get a super-resolved iris image on which the iris-code is computed [23]. This idea is further exploited and evaluated by Nguyen et al. [19] who introduce a quality driven fusion. Images have to be precisely registered in order to get ride of iris scale and rotation, otherwise important characteristics such as furrows and crypts are weakened.

The second family of methods, to which our algorithm belongs, tries to merge iris-codes obtained from a single image selected according to a quality criterion, in order to get one iris-code for a video. In their works, Hollingsworth et al. point out the existence of "fragile bits" in iris-code, i.e. bits which frequently switch between 0 and 1 . As these bits can degrade recognition performances, they propose to use in [9] a distance that takes into account the number of mutual fragile bits. This family of methods showed more promising than the previous, mainly because of mis-alignement in registering.

In this context, this paper proposes three main contributions:

1. A robust segmentation and an optimal tracking is proposed for the pupil detection.

2. The fusion of iris-codes is quality-driven by merging the score metrics proposed in [13] and in [25].

3. The algorithm runs in real-time (i.e 25 Frame Per Second (FPS)), by exploiting multi-thread decomposition of tasks and a smart selection of highest-quality iris images.

The evaluation of the proposed strategy is performed on the Multiple Biometric Grand Challenge (MBGC) portal dataset [21] using classical criteria: (i) ROC curve, (ii) Equal Error Rate (EER), (iii) False Rejection Rate (FRR) at low False Acceptance Rate (FAR), and (iv) top-rank histogram. We first study the influence of the number of merged iriscodes, and then the impact of masking more fragile bits. De- 


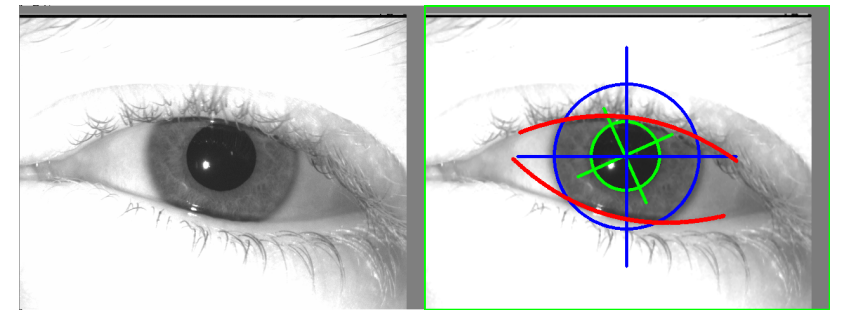

(a) Original image

(b) Pupil, iris and eyelids segmentation

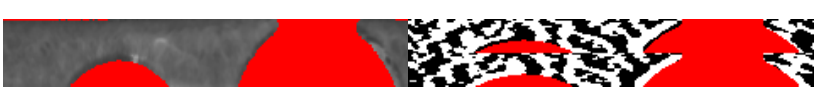

(c) Iris polar texture

(d) Iris-code

Fig. 1: Illustration of enrollment steps for iris coding.

scribed method is then compared to (i) the classical highestquality image selection approach [26], (ii) the quality-driven super-resolution method [19,20], (iii) the merged iris-code [8] and (iv) the merged iris-code with the "fragile bits distance" [9].

The remainder of the paper is organized as follows. Iris enrollment and fusion algorithms are presented in Section 2. A multi-threaded version of the entire algorithm to reach real-time processing is described in Section 3. Results of systematic experiments on the MBGC database are analysed in Section 4. Finally, conclusions and further works are sketched in Section 5.

\section{Quality-driven iris-code fusion}

The overall algorithm for segmenting pupil, selecting iris and merging iris-codes is sketched in [18]. The method consists of two main steps: the first one is dedicated to the iris enrollment procedure, and the second one is concerned with the iris-code fusion procedure and quality assessment.

\subsection{Iris enrollment}

\section{Pupil detection}

Usually, pupil is assumed to be a dark and homogeneous region of the image. To perform its segmenation and to reject false candidates, the following method is applied on a Region Of Interest (ROI), which is given by a Pairwise Kalman tracking (see section 4):

- As suggested by [15], the modes of the ROI histogram are detected using ROI-histogram minima, denoted $s_{i}$. Moreover, homogeneous regions are detected by adaptative thresholding.

- In order to reduce processing time, the following routine is used for the five lower histogram minima. The image is binarized according to $s_{i}$. The mask is then combined with the homogeneous regions. Hence, the smallest objects are deleted and closely linked regions separated by a morphological opening operation. Each remaining connex component of the image is labeled as a pupil candidate and its contour resampled to $P=64$ points. Following [6], a RANSAC algorithm is then used to determine the ellipse which best-fits the external contour. Our RANSAC algorithm makes use of the following parameters: a subsample of $P^{\prime}=32$ points, 50 RANSAC iterations, a distance threshold of $d_{\text {threshold }}=0.2 r^{\text {pupil }}$ and a valid point threshold of $P^{\prime \prime}=16$ where $r^{\text {pupil }}=$ $\left[\left[a^{\text {pupil }}\right]^{2}+\left[b^{\text {pupil }}\right]^{2}\right]^{\frac{1}{2}}$.

- The best-fitted region is selected as the last pupil candidate. If the mean distance between its ellipse and contour is too high $\left(d_{\text {threshold }}^{\prime}>0.1 r^{\text {pupil }}\right)$, then pupil segmentation is assumed failed.

\section{Iris localization and eyelid detection}

To find iris circle, we use J. Daugman's integro-differential operator [4] whose result is illustrated by the blue circle in Figure 1b. In order to segment eyelids or iris edges, we make use of a parabolic integro-differential operator, described in [12], using the parametric equation

$\left\{\begin{array}{l}x(t)=x_{i}+t \cos \theta+\left(a\left(t-x_{i}\right)^{2}+y_{0}\right) \sin \theta \\ y(t)=y_{i}-t \sin \theta+\left(a\left(t-x_{i}\right)^{2}+y_{0}\right) \cos \theta\end{array}\right.$

where $x_{i}, y_{i}, r_{i}$ are the iris circle coordinates and radius, $\theta$ varies from $-15^{\circ}$ to $15^{\circ}$ and from $165^{\circ}$ to $195^{\circ}$, a goes from 0 to $\left(2 r_{i}\right)^{-1}$ and $y_{0}$ goes from $r_{p}$ to $r_{i}$ for upper and lower eyelids. Eyelashes are detected using the same threshold as the one used for pupil detection.

\section{Iris-code computation}

Finally, the iris-code is mainly built according to Masek's algorithm [16]. First, the iris texture (i.e. the annular region between the pupil and the iris outer boundary) is unwrapped in the polar system, as illustrated in 1c. We set the resolution to classical values of 200 angular directions and 20 radii. Then the polar texture is filtered using 1D logGabor wavelets on each "circle". Following the Daugman's scheme, the complex-valued array is quantized according to phase-quadrants to get the so-called iris-code. An example is given in figure $1 \mathrm{~d}$ (red regions denote the occlusion mask).

\subsection{Iris-code fusion}

Here we intend to describe how fusion of iris-codes is performed and how fragile bits are detected. The fusion technique used in this work, inspirated from [8], takes several 


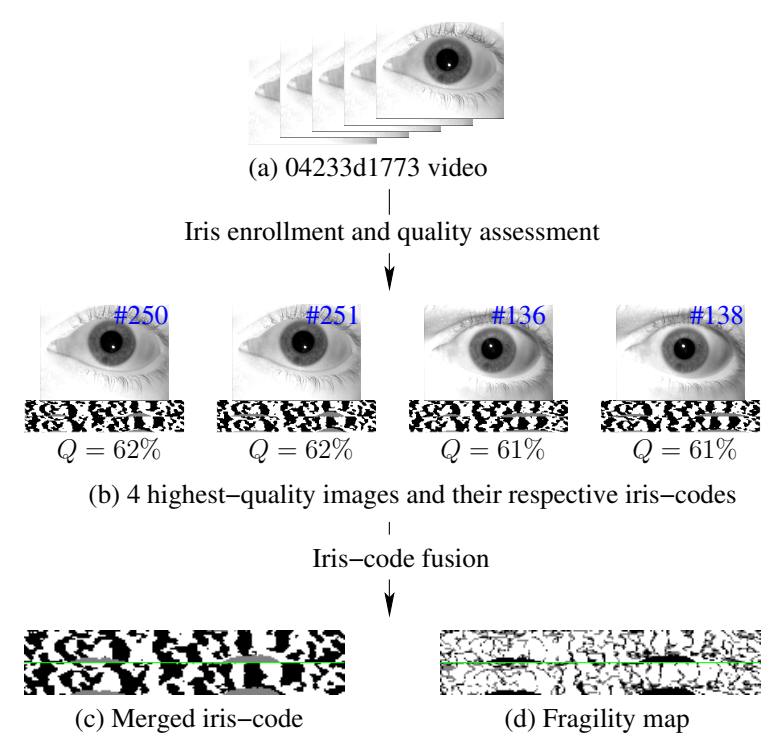

Fig. 2: Quality-fusion assessment scheme.

iris images and their corresponding iris-code as inputs. Fusion technique is composed of two main steps:

1. Image selection: for each image which passed iris enrollment, a focus score $Q_{\text {focus }}$ is computed using Kang and Park's metric [13]. This score is then multiplied by the usable iris texture area ( $c f$. [25]), which gives the score $Q_{\text {score }}$. The fusion is performed on the $P$ highestscored iris-codes. Figure $2 \mathrm{~b}$ illustrates the selection.

2. Iris-code fusion and detection of fragile bits: The iriscodes are registered with the highest-scored iris-code and then their weighted $Q_{\text {score }}$-mean is computed. It gives a "fuzzy", intensity-valued iris-code $\hat{I}(r, \theta)$. Finally, a classical binary iris-code is obtained by thresholding $\hat{I}(r, \theta)$ with respect to 0.5 , and its associated fragility map is computed according to

$$
f(r, \theta)=1-2 \times|\hat{I}(r, \theta)-0.5|
$$

Binary iris-code and fragility map for the MBGC video \#04233d1773 are respectively given in figures $2 \mathrm{c}$ and $2 \mathrm{~d}$.

\section{3 "Real-time" iris template processing}

This section is intended to desbribe a "real-time" version of the iris enrollment algorithm described in Section 2. Three techniques are combined to achieve the goal : First, an optimal Kalman-like tracking algorithm follows the pupil. Second, a smart selection of high quality images reduces the number of frames to be enrolled. Third, segmentation tasks are decomposed into four threads to divide processing time.

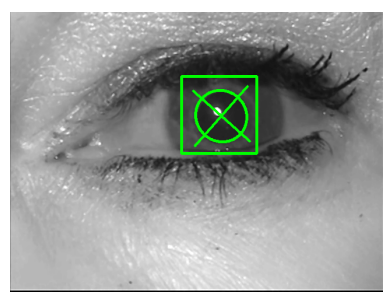

Fig. 3: Example of pupil ROI from the MBGC 04470d1628 video.

\subsection{Pairwise Kalman tracking}

To predict pupil positions and radii in a video, the following Kalman-like system of equations is used

$\underbrace{\left(\begin{array}{c}\boldsymbol{x}_{n+1} \\ \boldsymbol{z}_{n}\end{array}\right)}_{\boldsymbol{t}_{n+1}}=\underbrace{\left(\begin{array}{cc}\boldsymbol{F}^{x, x} & \boldsymbol{F}^{x, z} \\ \boldsymbol{I}^{z, z} & \mathbf{0}\end{array}\right)}_{\boldsymbol{F}} \underbrace{\left(\begin{array}{c}\boldsymbol{x}_{n} \\ \boldsymbol{z}_{n-1}\end{array}\right)}_{\boldsymbol{t}_{n}}+\underbrace{\left(\begin{array}{c}\boldsymbol{\omega}_{n+1}^{x} \\ \boldsymbol{\omega}_{n+1}^{z}\end{array}\right)}_{\boldsymbol{\omega}_{n+1}}$

where

$$
\boldsymbol{x}_{n}=\left(\begin{array}{c}
x_{n}^{\text {pupil }}-w / 2 \\
y_{n}^{\text {pupil }}-h / 2 \\
r_{n}^{\text {pupil }}
\end{array}\right)
$$

with $w$ and $h$ represent respectively the width and the height of the image and $\boldsymbol{\omega}_{n+1}$ and where $\boldsymbol{t}_{0}$ are respectively distributed according to two Gaussian laws $\mathcal{N}\left(0, \boldsymbol{Q}=\left(\begin{array}{cc}\boldsymbol{Q}^{x, x} & \mathbf{0} \\ \mathbf{0} & \mathbf{0}\end{array}\right)\right)$ and $\mathcal{N}\left(\hat{\boldsymbol{t}}_{0}, \boldsymbol{Q}_{0}\right)$ corresponds to the hidden states, and $\boldsymbol{z}_{n}$ to the observed states. Observations are given by the pupil segmentation algorithm. In fact, this model is more general that the classical Kalman system, and belongs to the family of Pairwise Kalman Filter (PKF), see [5,2] for details. Exact and robust prediction and filtering equations are reported in [17].

Let us denote $\hat{\boldsymbol{x}}_{n \mid n-1}=\left(\hat{x}_{n \mid n-1}^{\text {pupil }}, \hat{y}_{n \mid n-1}^{\text {pupil }}, \hat{r}_{n \mid n-1}^{\text {pupil }}\right)^{T}$ the expectations and $\sigma_{n \mid n-1}\left(x^{\text {pupil }}\right), \sigma_{n \mid n-1}\left(y^{\text {pupil }}\right), \sigma_{n \mid n-1}\left(r^{\text {pupil }}\right)$ the standard deviations of the predicted position and radius. The rectangular Region Of Interest (ROI), in which the pupil is searched for, is defined by:

$$
\left\{\begin{array}{l}
x_{R O I}=\hat{x}_{n \mid n-1}^{\text {pupil }}-\frac{w_{R O I}}{2} \\
y_{R O I}=\hat{y}_{n \mid n-1}^{\text {pupil }}-\frac{h_{R O I}}{2} \\
w_{R O I}=2 \hat{r}_{n \mid n-1}^{\text {pupil }}+N\left[\sigma_{n \mid n-1}\left(x^{\text {pupil }}\right)+\sigma_{n \mid n-1}\left(r^{\text {pupil }}\right)\right] \\
h_{R O I}=2 \hat{r}_{n \mid n-1}^{\text {pupil }}+N\left[\sigma_{n \mid n-1}\left(y^{\text {pupil }}\right)+\sigma_{n \mid n-1}\left(r^{\text {pupil }}\right)\right]
\end{array}\right.
$$

where $N$ is a parameter set to 4 in our experiments. An example of ROI is drawn in green in Figure 3.

The model (3) is parameterized by matrices $\boldsymbol{F}, \boldsymbol{Q}$, which are difficult to set manually. So, we make use of an automatic learning algorithm [17] on the MBGC video \#02463d1980. 
Table 1: Pairwise Kalman parameters learned from one video sequence.

\begin{tabular}{|l|c|}
\cline { 2 - 3 } \multicolumn{1}{c|}{} & $\hat{\boldsymbol{\theta}}^{(E M)}$ \\
\hline $\boldsymbol{F}^{x, x}$ & $\left(\begin{array}{ccc}1.59 & 0.08 & -0.25 \\
0.05 & 1.31 & -0.17 \\
-0.04 & 0.15 & 0.62\end{array}\right)$ \\
\hline \multirow{3}{*}{$\boldsymbol{F}^{x, z}$} & $\left(\begin{array}{ccc}-0.61 & -0.07 & 0.20 \\
-0.06 & -0.32 & 0.11 \\
0.02 & -0.15 & 0.26\end{array}\right)$ \\
\hline \multirow{2}{*}{$\boldsymbol{Q}^{x, x}$} & $\left(\begin{array}{ccc}5.91 & 0.45 & 2.22 \\
0.45 & 9.68 & 2.95 \\
2.22 & 2.95 & 10.55\end{array}\right)$ \\
\hline
\end{tabular}

The so-estimated parameters are reported in Tab 1 and reused for all MBGC videos. The model seems to fit well data since values in process covariance matrix $Q^{x, x}$ are small with respect to images size $(640 \times 480$ for MBGC dataset $)$.

During hocking, pupils are frequently lost due to out of focus, closed eyelids... So, the model has to take into account the skipped frames to predict the ROI at frame $n+p$ from valid pupil data at frame $n$. Skipping $p$ frames is equivalent to replace $\boldsymbol{F}$ and $\boldsymbol{Q}$ parameters in system (3) by:

$\boldsymbol{F}_{p}^{\prime}=\boldsymbol{F}^{p+1}$,

$\boldsymbol{Q}_{p}^{\prime}=\sum_{r=0}^{p} \boldsymbol{F}^{r} \boldsymbol{Q}\left[\boldsymbol{F}^{r}\right]^{T}$,

see Appendix A.

Tracking can significantly reduce segmentation times. Indeed, using a $2.3 \mathrm{Ghz}$ dual-core $\mathrm{i} 3$ processor, pupil segmentation takes about $100 \mathrm{~ms}$ without tracking and about $55 \mathrm{~ms}$ with tracking.

\subsection{Smart selection and multi-threading algorithm}

The algorithm described in Section 2 is suitable for parallelization with the help of a smart selection of images, according to the following four tasks:

1. Acquistion thread: The first thread acquires frame from the video stream or capture device and computes the focus score $Q_{\text {focus }}$. Acquired images are then buffered for being exploited by the second thread. This thread is blocked to $f$ FPS.

2. Pupil thread: The second thread, depicted in Figure 4a, retrieves buffered images to segment and track the pupils. If the segmentation has succeeded, image and pupil characteristics are buffered for being exploited by the third thread.

3. Iris thread: The third thread, illustrated on Figure $4 b$, retrieves buffered data to localize iris, eyelids, eyelashes, spots, and finally to construct the iris-code. If the quality

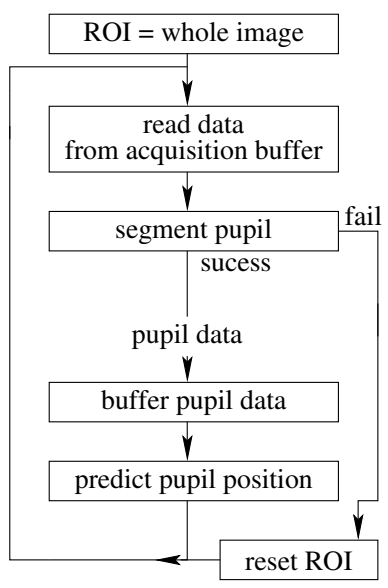

(a) Pupil thread

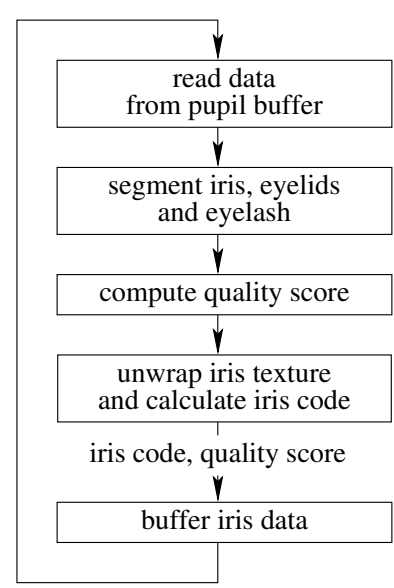

(b) Iris thread
Fig. 4: Flowcharts of the two main threads for the iris enrollment.

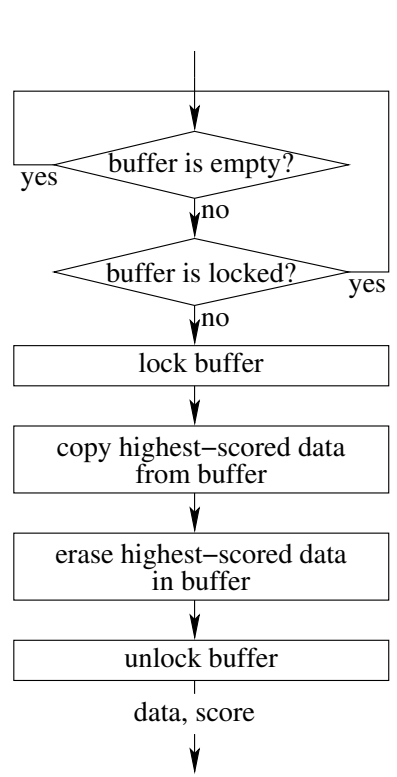

(a) Buffer reading

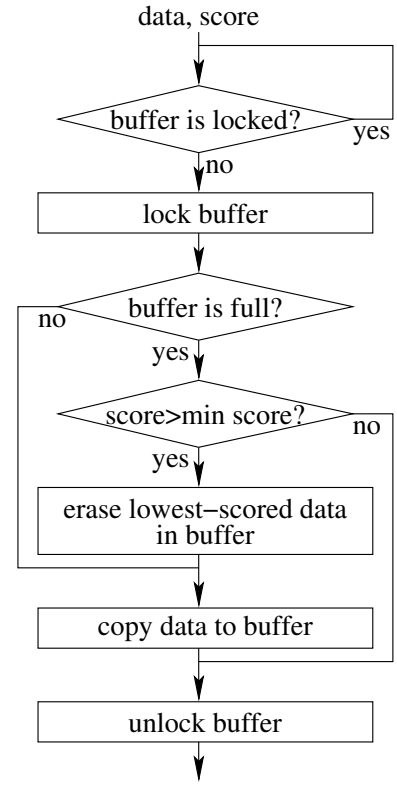

(b) Buffer writting
Fig. 5: Flowcharts of buffer running modes.

score $Q_{\text {score }}$ is above a given threshold, then the iriscode is buffered for its exploitation by the fourth thread.

4. Fusion thread: The last thread fuses the highest-quality iris-codes, according to $Q_{\text {score }}$ in order to obtain the fragility map $2 \mathrm{c}$ and merged iris-code $2 \mathrm{~d}$.

For acquistion and pupil threads, the buffers are sorted according to $Q_{\text {focus }}$ whereas the iris thread is sorted with respect to $Q_{\text {score }}$. As the strategy is clamped by the buffer capacities, each new entry is sorted according to its score and only highest-quality images are selected for enrollment. Figure 5 sketches the writting and reading modes for each buffer. 
When FPS is locked to $f=25$, about $75 \%$ of the pupils are detected and about $15 \%$ of the irises are enrolled. When using sequential method (i.e. without buffer), only $13 \%$ of pupils and irises are segmented, unrelated to theirs quality or focus scores.

\section{Performance evaluation on MBGC database}

Iris verification experiments have been conducted on the MBGC dataset [21]. This dataset consists of 986 close-up eye videos and 8589 NIR iris still-images of 137 individuals. Iris videos and individual images are segmented and merged using the algorithm described in Sections 2 and 3. The still-image dataset is used to enroll and label each individual, whereas the video dataset is used to test the recognition performance of the proposed method against the image dataset.

The method presented above has succeeded to enroll 99.8\% of videos ${ }^{1}$ and all iris classes (each iris class contains at least one image, but most classes contain more than 10 images). Regarding the still-image dataset, $96.7 \%$ of images were enrolled. We verified visually that defeated images are either severely occluded or very blurred or very poor-contrasted.

Once both datasets are enrolled, we matched iris templates from video dataset to ones from still-image dataset using Hamming distance. The results are saved in the form of Detection Error Trade-off (DET) plots and top-rank histograms and evaluated by 2 criteria: Equal Error Rate (EER) and False Rejection Rate (FRR) when the False Acceptance Rate (FAR) is set to $10^{-6}$.

Two experiments were done to estimate the influence of the number of merged iris-codes and to determine the impact of masking fragile bits on recognition performances. Finally, these results were compared with three state-of-theart methods: one based on the iris texture fusion and two other ones based on iris-code fusion without score assessment.

\subsection{Influence of the number of merged iris-codes}

The first experiment has been conducted to determine (1) if there is some interest in merging iris-codes, and, if yes, (2) if there is an optimum number of iris-codes to merge before performances start to degrade. Indeed, as the fusion includes more and more images, and since we consider images in their scoring order, the fusion process adds lower quality images. To verify these points, we applied the fusion technique with a number of merged iris-codes varying from 1 to 30 frames. The distribution of imposture and genuine distances are reported in Figure 6 for 1, 5, 10 and 30 merged iris-codes. The top-ranks are given in Figure 7 whereas ROC

\footnotetext{
1 Only videos \#05344v27 and \#05416v25 failed to enroll.
}

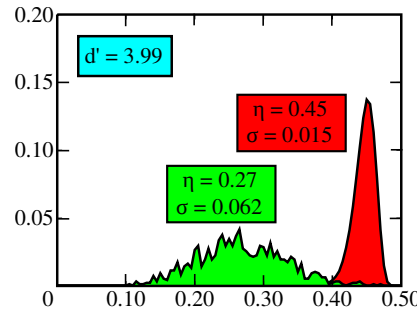

(a) Highest-quality image

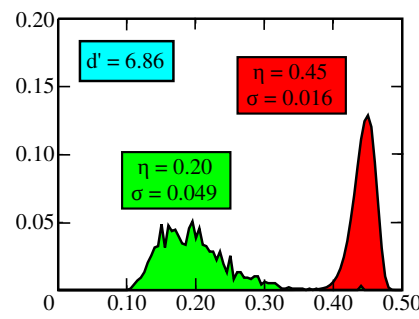

(c) 10 images

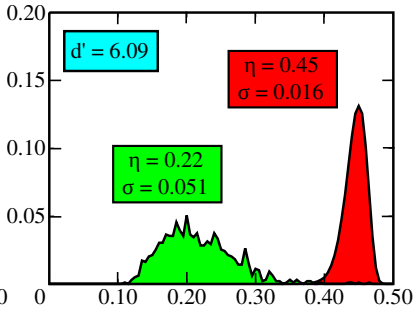

(b) 5 images

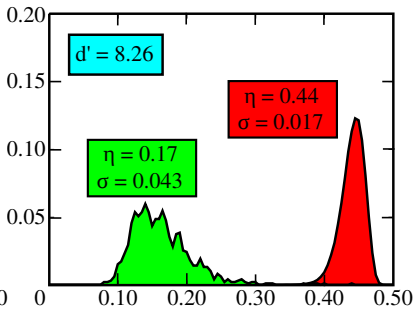

(d) 30 images
Fig. 6: Distance distributions for the highest quality image (a), the fusion of 5 (b), of 10 (c) and of 30 (d) highest-quality images. Impostor distance distributions are in red, genius distance distributions are in green. $\eta, \sigma$ and $d^{\prime}$ report the estimated mean, standard deviation of distributions and the statistical metric between distributions $d^{\prime}=\frac{\left|\eta_{g}-\eta_{i}\right|}{\sqrt{0.5\left(\sigma_{i}^{2}+\sigma_{g}^{2}\right)}}$.

curves and EER and FRR at FAR $=10^{-6}$ curves are reported in Figures 8 and 9 respectively.

The keypoint is that all indicators confirm an improvement of recognition performances when the number of merged iris-codes goes from 1 to 10 . Regarding distance distributions, the separation between genius and impostor distributions increases when the number of merged images increases. As seen in the top-rank histograms, irises are better classified when the number of merged images increases. Moreover, the FRR at FAR $=10^{-6}$ continues to decrease when merging beyond 10 iris-codes. Finally, the fusion of several frames outperforms the use of the only highest-quality image: EER decreases from $1.58 \%$ to $0.2 \%$ (12 images), and FFR at FAR $=10^{-6}$ decreases from $40 \%$ to $0.8 \%$ (22 images). In conclusion, merging a large number of frames can have a real interest for high-security applications.

\subsection{Influence of fragile bits}

The second experiment was conducted to determine the impact of masking fragile bits on iris recognition performances, using the fragility map ( $P \%$ of most fragile bits are masked and ignored during the hamming distance computation). Masking more and more fragile bits reduces the information on iris texture taking into account by iris-code and so can deteriorate performances. Distance distributions for Fragile Bit 


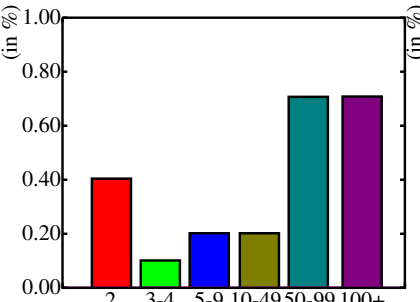

(a) Highest-quality image

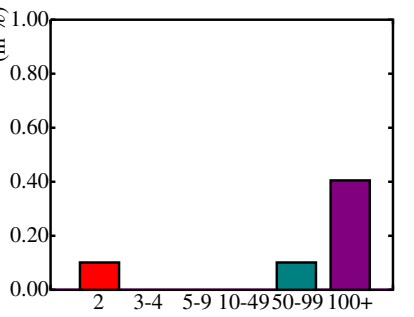

(b) 5 images

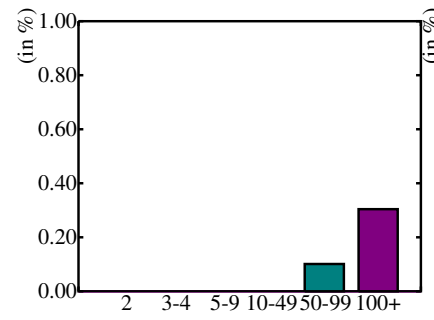

(c) 10 images

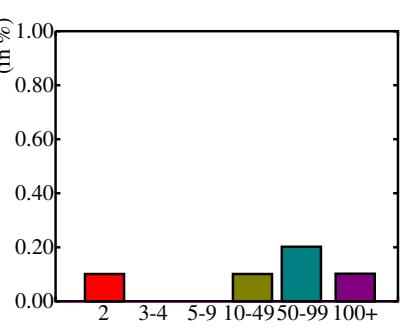

(d) 30 images
Fig. 7: Rank of true class when classification have failed for the highest-quality image (a), the fusion of 5 (b), of 10 (c) and of $30(d)$ highest-quality iris-codes.

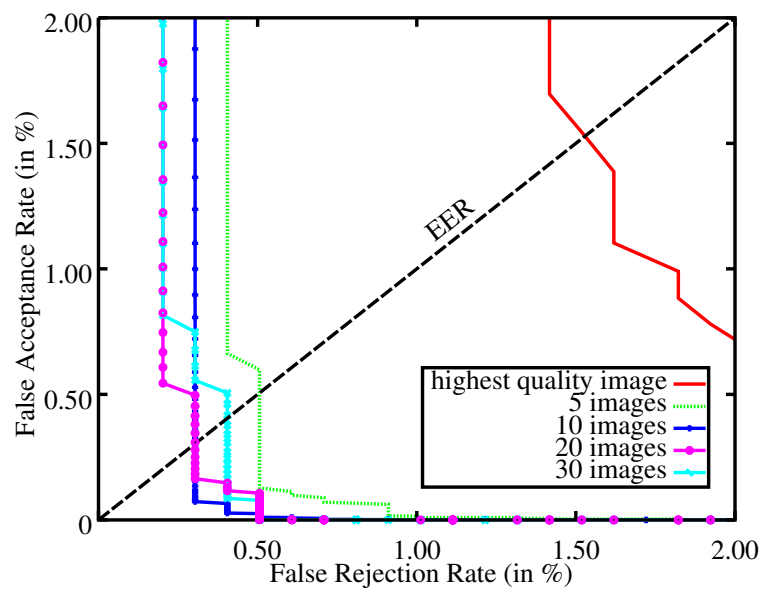

Fig. 8: DET plots: influence of the number of merged iriscodes on recognition performances.

Rates (FBR) of $0 \%, 15 \%, 30 \%$ and $45 \%$ are reported in Figure 10 when using the 10 highest-quality images. The evolution of EER and FRR at FAR $=10^{-6}$ for different numbers of merged iris-codes is shown in Figure 11.

Globally, merging 10 highest-quality iris-code always gives the best EER whatever the FBR. The best EER (0.2\%) is reached when merging 10 iris-codes from a FBR of $20 \%$, and when fusing above 20 iris-codes from a FBR of $30 \%$. Globally, merging more and more iris-codes improves FRR at $\mathrm{FAR}=10^{-6}$ whatever the FBR. It can be pointed out that the best FFR at FAR $=10^{-6}$ is obtained only when merging above 20 iris-codes for a $20 \%$-FBR. Regarding the distance distributions, masking fragile bits tends to reduce the genius

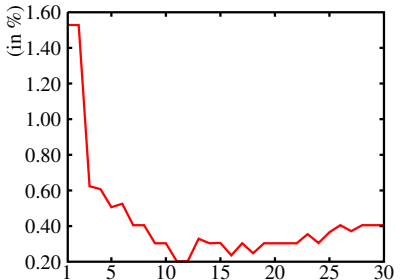

(a) EER

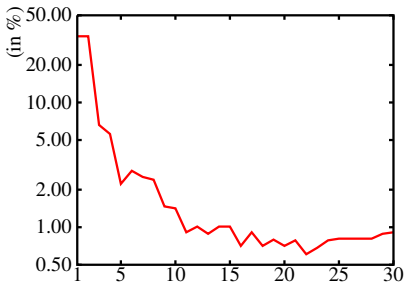

(b) FAR $=10^{-6}(\log$ scale)
Fig. 9: Evolution of EER (a) and FRR at FAR $=10^{-6}$ (b) with respect to the number of merged iris-codes.

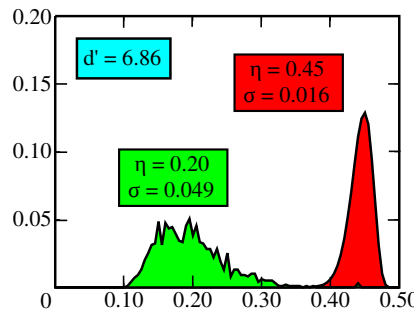

(a) FBR of $0 \%$

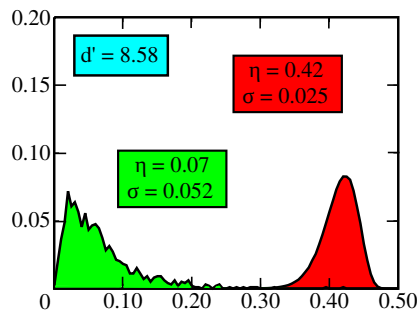

(c) FBR of $30 \%$

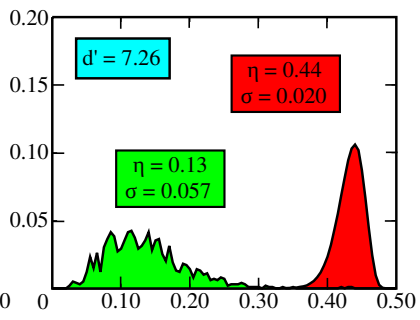

(b) FBR of $15 \%$

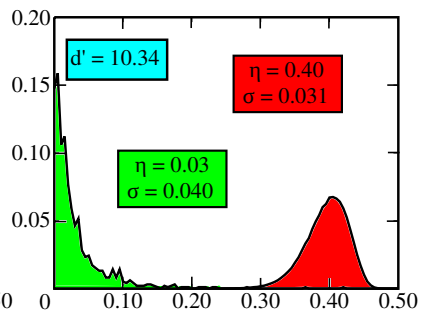

(d) FBR of $45 \%$

Fig. 10: Distance distributions for the fusion of 10 iris-codes with different Fragile Bit Rates.

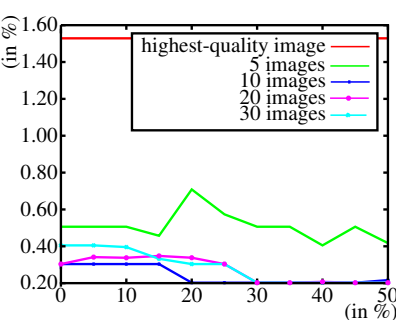

(a) EER

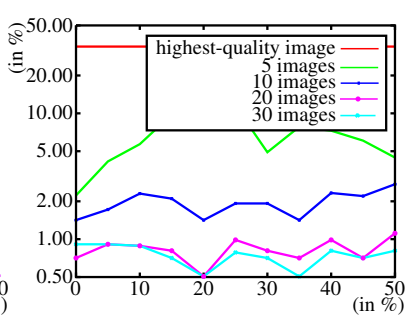

(b) FRR at FAR $=10^{-6}$ (log scale)

Fig. 11: Evolution of EER (a) and FRR at FAR $=10^{-6}$ (b) with respect to the FBR.

distance mean and covariance, and to increase variance of imposture distances.

So, two main conclusions can be drawn from the experiment on the MBGC datasets: 
Table 2: Comparison of EERs obtained on MBGC iris datadry.

\begin{tabular}{|c|c|c|c|}
\hline$[19]$ & {$[20]$} & {$[8]$} & {$[9](20$ radii $)$} \\
\hline$\sim 0.80 \%$ & $\sim 0.50 \%$ & $\sim 0.87 \%$ & $\sim 0.80 \%$ \\
\hline$[9](10$ radii $)$ & this work (20 radii) \\
\hline \multicolumn{2}{|c|}{$\sim 0.29$} & $\mathbf{0 . 2 0} \%$ \\
\hline
\end{tabular}

1. for large-public applications, it is advisable to merge about 10 iris-codes with a fragile bit rate of $20 \%$.

2. For high-security applications, it is recommended to merge more than 20 iris-codes with a fragile bit rate of $20 \%$.

\subsection{Comparison with other fusion techniques}

The method presented here was compared to four other studies

- The first paper [19] depictes a super-resolution iris texture method to improve recognition performances.

- The second one [20] proposes a super-resolution method of iris features computed from 2D-Gabor wavelets.

- The third one [8] proposes to merge iris-codes without quality assessment and to mask the frequently changing bits.

- The fourth one [9] makes use of a fragile bit distance, which improves third paper results.

Table 2 reports EER for the best results obtained from the four methods and our algorithm. Our method shows a drastic reduction of the EER.

\section{Conclusion}

In this paper, we proposed a "real-time" quality-based iriscode fusion algorithm to improve iris recognition performance from close-up eye videos. Real-time processing (i.e. 25 FPS) was achevied by multi-thread decomposition of the processing chain and a quality-based selection of frames to be further processed by the threads. A fragility map was assiocated to each iris-code, which measures the confidence of each bits to be 0 or to be 1 .

The algorithm was systematically evaluated on the MBGC database [21] with respect to the number of merged iriscodes and the influence of the fragile bit rate. The proposed fusion process improves performances significantly. Moreover, the adequate parameter values for high-security and large-public applications have been identified. Performances have also been compared with three competitive methods and our algorithm divides the EER by 1.5 to 4 .
The log-Gabor wavelets were choosen for then high performances, widespread and low complexity. Nevertheless, higher performances could have been obtained by using biorthogonal wavelets $[24,1]$. Moreover, the Hamming distance can be replaced by another matching criteria [3]. Also, the RANSAC based algorithm used to fit the pupil with an ellipse proved to be very efficient; a comparison with a recently published snake-based method [14] is of great interest.

Acknowledgements Authors would like to thank DGA (French $D i$ rection Générale de l'Armement) and CNRS for financial support.

\section{A Proof of equations (5) and (6)}

Let them prove by recurrence:

- For $p=0$, the proof is trivial $\left(\boldsymbol{F}_{0}^{\prime}=\boldsymbol{F}\right.$ and $\left.\boldsymbol{Q}_{0}^{\prime}=\boldsymbol{Q}^{\prime}\right)$

- Let assume (5) and (6) true for $p-1$. Then,

$\boldsymbol{t}_{n+p}=\boldsymbol{F}_{p-1}^{\prime} \boldsymbol{t}_{n}+\boldsymbol{\omega}_{n, p-1}$

where $\boldsymbol{\omega}_{n, p-1}$ follow the Gaussian law $\mathcal{N}\left(0, Q_{p-1}^{\prime}\right)$. Let them prove for $p$ :

$$
\begin{aligned}
& \text { Proof } \\
& \boldsymbol{t}_{n+p+1}=\boldsymbol{F} \boldsymbol{F}_{p-1}^{\prime} \boldsymbol{t}_{n}+\boldsymbol{F} \underbrace{\boldsymbol{\omega}_{n, p-1}}_{\sim \mathcal{N}\left(0, Q_{p-1}^{\prime}\right)}+\underbrace{\boldsymbol{\omega}_{n+p+1}}_{\sim \mathcal{N}(0, Q)} \\
& =\boldsymbol{F}^{p+1} \boldsymbol{t}_{n}+\underbrace{\boldsymbol{F} \boldsymbol{\omega}_{n, p-1}}_{\sim \mathcal{N}\left(0, \boldsymbol{F} Q_{p}^{\prime} \boldsymbol{F}^{T}\right)}+\underbrace{\boldsymbol{\omega}_{n+p+1}}_{\sim \mathcal{N}(0, Q)} \\
& =\boldsymbol{F}_{p}^{\prime} \boldsymbol{t}_{n}+\underbrace{\boldsymbol{F} \boldsymbol{\omega}_{n, p-1}}_{\sim \mathcal{N}\left(0, \boldsymbol{F}\left[\sum_{r=0}^{p-1} \boldsymbol{F}^{r} \boldsymbol{Q}\left[\boldsymbol{F}^{r}\right]^{T}\right] \boldsymbol{F}^{T}\right)}+\underbrace{\boldsymbol{\omega}_{n+p+1}}_{\sim \mathcal{N}(0, Q)} \\
& =\boldsymbol{F}_{p}^{\prime} \boldsymbol{t}_{n}+\underbrace{\boldsymbol{F} \boldsymbol{\omega}_{n, p-1}}_{\sim \mathcal{N}\left(0, \sum_{r=1}^{p} \boldsymbol{F}^{r} \boldsymbol{Q}\left[\boldsymbol{F}^{r}\right]^{T}\right)}+\underbrace{\boldsymbol{\omega}_{n+p+1}}_{\sim \mathcal{N}(0, Q)} \\
& =\boldsymbol{F}_{p}^{\prime} \boldsymbol{t}_{n}+\underbrace{\boldsymbol{F} \boldsymbol{\omega}_{n, p-1}+\boldsymbol{\omega}_{n+p+1}}_{\sim \mathcal{N}\left(0, \sum_{r=0}^{p} \boldsymbol{F}^{r} \boldsymbol{Q}\left[\boldsymbol{F}^{r}\right]^{T}\right)} \\
& =\boldsymbol{F}_{p}^{\prime} \boldsymbol{t}_{n}+\underbrace{\boldsymbol{\omega}_{n, p+1}}_{\sim \mathcal{N}\left(0, Q_{p}^{\prime}\right)}
\end{aligned}
$$

\section{References}

1. Abhyankar, A., Schuckers, S.: Iris quality assessment and bi-orthogonal wavelet based encoding for recognition. Pattern Recognition 42(9), 1878 - 1894 (2009). DOI http://dx.doi.org/10.1016/j.patcog.2009.01.004. URL http://www.sciencedirect.com/science/ article/pii/s0031320309000053

2. Ait-el-Fquih, B., Desbouvries, F.: Kalman filtering in triplet Markov chains. IEEE Trans. on Signal Processing 54(8), 29572963 (2006)

3. Costa Filho, C., Pinheiro, C., Costa, M., Albuquerque Pereira, W.: Applying a novelty filter as a matching criterion to iris recognition for binary and real-valued feature vectors. Signal, Image and Video Processing 7(2), 287-296 (2013). DOI 10. 1007/s11760-011-0237-5. URL http://dx.doi.org/10. $1007 /$ s11760-011-0237-5 
4. Daugman, J.: How iris recognition works. IEEE Trans. on Circuits and Systems for Video Technology 14(1), 21-30 (2004). DOI 10.1109/TCSVT.2003.818350

5. Desbouvries, F., Pieczynski, W.: Triplet Markov models and Kalman filtering. Comptes Rendus de l'Académie des Sciences - Mathématique - Série I 336(8), 667-670 (2003)

6. Fischler, M.A., Bolles, R.C.: Random sample consensus: a paradigm for model fitting with applications to image analysis and automated cartography. Commun. ACM 24(6), 381-395 (1981). DOI $10.1145 / 358669.358692$

7. Ghodrati, H., Dehghani, M., Danyali, H.: A new accurate noiseremoving approach for non-cooperative iris recognition. Signal, Image and Video Processing 8(1), 1-10 (2014). URL http: // dx.doi.org/10.1007/s11760-012-0396-z

8. Hollingsworth, K., Ortiz, E., Bowyer, K.: The best bits in an iris code. IEEE Trans. on Pattern Analysis and Machine Intelligence 31(6), 964-973 (2009). DOI 10.1109/TPAMI.2008.185

9. Hollingsworth, K., Ortiz, E., Bowyer, K.: Improved iris recognition through fusion of Hamming distance and fragile bit distance. IEEE Trans. on Pattern Analysis and Machine Intelligence 33(12), 2465-2476 (2011). DOI 10.1109/TPAMI.2011.89

10. Jain, A., Ross, A., Nandakumar, K.: Introduction to Biometrics. SpringerLink : Bücher. Springer US (2011)

11. Jain, A., Ross, A., Prabhakar, S.: An introduction to biometric recognition. IEEE Trans. on Circuits and Systems for Video Technology 14(1), 4-20 (2004). DOI 10.1109/TCSVT.2003.818349

12. Jang, Y.K., Kang, B.J., Park, K.R.: A study on eyelid localization considering image focus for iris recognition. Pattern Recognition Letters 29(11), 1698-1704 (2008). DOI 10.1016/j.patrec.2008.05. 001

13. Kang, B., Park, K.: A study on iris image restoration. In: T. Kanade, A. Jain, N. Ratha (eds.) Audio- and Video-Based Biometric Person Authentication, Lecture Notes in Computer Science, vol. 3546, pp. 31-40. Springer Berlin Heidelberg (2005). DOI $10.1007 / 11527923 \backslash 44$

14. Lefevre, T., Dorizzi, B., Garcia-Salicetti, S., Lemperiere, N., Belardi, S.: Effective elliptic fitting for iris normalization. Computer Vision and Image Understanding 117(6), 732 - 745 (2013). DOI http://dx.doi.org/10.1016/j.cviu.2013.01.005

15. Li, P., Liu, X., Xiao, L., Song, Q.: Robust and accurate iris segmentation in very noisy iris images. Image and Vision Computing 28(2), 246 - 253 (2010). DOI http://dx.doi.org/10.1016/j.imavis. 2009.04.010

16. Masek, L.: Recognition of human iris patterns for biometric identification. Tech. rep., University of Western Australia (2003)

17. Némesin, V., Derrode, S.: Robust blind pairwise Kalman algorithms using QR decompositions. IEEE Trans. on Signal Processing 61(1), 5-9 (2013)

18. Némesin, V., Derrode, S., Benazza-Benyahia, A.: Gradual iris code construction from close-up eye video. In: J. Blanc-Talon, W. Philips, D.C. Popescu, P. Scheunders, P. Zemcík (eds.) 14th Int. Conf. on Advanced Concepts for Intelligent Vision Systems (ACIVS'12), Lecture Notes in Computer Science, vol. 7517, pp. 12-23. Springer (2012)

19. Nguyen, K., Fookes, C., Sridharan, S., Denman, S.: Quality-driven super-resolution for less constrained iris recognition at a distance and on the move. IEEE Trans. on Information Forensics and Security 6(4), 1248-1258 (2011). DOI 10.1109/TIFS.2011.2159597

20. Nguyen, K., Fookes, C., Sridharan, S., Denman, S.: Featuredomain super-resolution for iris recognition. Computer Vision and Image Understanding 117(10), 1526 - 1535 (2013). DOI http://dx.doi.org/10.1016/j.cviu.2013.06.010. URL http://www.sciencedirect.com/science/ article/pii/S1077314213001306

21. Phillips, P.J., Flynn, P.J., Beveridge, J.R., Scruggs, W.T., O’ Toole, A.J., Bolme, D., Bowyer, K.W., Draper, B.A., Givens, G.H., Lui, Y.M., Sahibzada, H., Scallan, J.A., Weimer, S.: Overview of the Multiple Biometrics Grand Challenge. In: Proc. of the 3rd Int. Conf. on Advances in Biometrics, (ICB'09), pp. 705714. Springer-Verlag, Berlin, Heidelberg (2009). DOI 10.1007/ 978-3-642-01793-3\-72

22. Roy, K., Bhattacharya, P., Suen, C.: Iris segmentation using game theory. Signal, Image and Video Processing 6(2), 301-315 (2012). URL http://dx.doi.org/10.1007/ s11760-010-0193-5

23. Shin, K.Y., Park, K.R., Kang, B.J., Park, S.J.: Super-resolution method based on multiple multi-layer perceptrons for iris recognition. In: Proc. of the 4th Int. Conf. on Ubiquitous Information Technologies Applications (ICUT'09), pp. 1-5 (2009). DOI 10.1109/ICUT.2009.5405701

24. Szewczyk, R., Grabowski, K., Napieralska, M., Sankowski, W., Zubert, M., Napieralski, A.: A reliable iris recognition algorithm based on reverse biorthogonal wavelet transform. Pattern Recognition Letters 33(8), 1019 - 1026 (2012). DOI http://dx.doi.org/ 10.1016/j.patrec.2011.08.018

25. Tabassi, E., Grother, P., Salamon, W.: IREX II - IQCE - Iris Quality Calibration and Evaluation. Tech. rep., NIST Interagency Report $7820(2011)$

26. Yooyoung, L., Micheals, R.J., Phillips, P.J.: Improvements in video-based automated system for iris recognition (VASIR). In: Workshop on Motion and Video Computing (WMVC'09), pp. 18 (2009). DOI 10.1109/WMVC.2009.5399237 